\title{
Técnicas intervencionistas radioguiadas para tratamiento del hombro doloroso
}

\author{
J.M. Orduña Valls ${ }^{1}$, C.L. Nebreda Clavo $^{1}$, A. Ojeda Niño ${ }^{1,2}$, L. Aliaga Font ${ }^{1}$ y R. Vallejo Salamanca ${ }^{1,3}$ \\ ${ }^{1}$ Clínica del Dolor. Centro Médico Teknon. Barcelona. ${ }^{2}$ Hospital Clinic. Barcelona. ${ }^{3}$ Millenium Pain \\ Management. Bloomington, Illinois. EE.UU.
}

Orduña Valls JM, Nebreda Clavo CL, Ojeda Niño A, Aliaga Font L, Vallejo Salamanca R. Técnicas intervencionistas radioguiadas para tratamiento del hombro doloroso. Rev Soc Esp Dolor 2014; 21(2): 112-117.

\section{INTRODUCCIÓN}

El cíngulo del hombro está compuesto por cuatro articulaciones específicas y una general: esternoclavicular, acromioclavicular, glenohumeral, subacromial y articulación escapulotorácica. Para el buen funcionamiento de la articulación es fundamental el sincrónico funcionamiento de todas ellas (1). La incidencia de dolor en esta articulación es alta, estimándose en 24 de cada 1.000 pacientes de Atención Primaria, con una mayor tendencia entre mujeres (60 \%) (1). Es importante resaltar la alta tendencia a la recidiva a pesar del carácter habitualmente autolimitado (con $60 \%$ de recuperación pasado un año) $(2,3)$.

Dentro de la etiología que conduce al desarrollo de este proceso doloroso es poco habitual encontrar una única causa como responsable del proceso (más allá de procesos traumáticos eventuales), siendo frecuente la coexistencia de patologías cuyo reconocimiento será de vital importancia para el correcto tratamiento. Entre las más frecuentes se encuentran procesos inflamatorios de la membrana sinovial (de carácter aséptico), inflamación de las articulaciones acromioclavicular, esternoclavicular y glenohumeral; asociado en un alto número de casos a un síndrome miofascial y cierto grado de afectación del manguito de los rotadores.

Recibido: 15-10-2013.

Aceptado: 20-12-2013
La coexistencia de estas alteraciones podrá explicar el proceso doloroso multifactorial que habitualmente encontramos en estos pacientes. Es importante determinar la coexistencia de los diferentes factores con el fin de tratarlos además de realizar una evaluación sistemática de la columna cervical, así como de la torácica, con el fin de descartar patología que pudiera manifestarse a este nivel.

\section{ANATOMÍA}

El hombro es la articulación con mayor rango de movimiento del cuerpo humano; es en realidad un conjunto de articulaciones cuyo funcionamiento individual es fundamental para que no se vea afectado el conjunto. El acromion, la coracoides y el ligamento coracoacromial forman el arco coracoacromial, que junto a la espina escapular y la articulación acromioclavicular forman el desfiladero del supraespinoso. Este es el lugar por el que discurrirá el manguito de los rotadores en íntima relación con las bursas subacromial y subdeltoidea. El tendón de la porción larga del bíceps podrá ser considerado como integrante del manguito desde el punto de vista funcional (no así desde el anatómico) debido a su acción depresora y de estabilización de la cabeza humeral (4-6).

\section{DIAGNÓSTICO}

Es fundamental una correcta anamnesis y exploración física con el fin de diferenciar los distintos factores influyentes en el proceso. El síntoma más frecuente en la historia clínica es la presencia de dolor al apoyarse sobre la extremidad afecta (más frecuente de noche), así como cierto grado de limitación funcional. La localización y el 
patrón de irradiación pueden aportar más pistas acerca del origen (7). Otras condiciones importantes que considerar y descartar son la presencia de fiebre, pérdida de peso, disnea y angina de pecho, cuya presencia obligaría a descartar patologías como eventos isquémicos miocárdicos, procesos tumorales (Pancoast), etc.

\section{Exploración física}

La exploración de la articulación se iniciará con la inspección de ambos hombros, de forma segmentaria y comparativa, para continuar con la palpación de las estructuras óseas y tejidos blandos que la conforman. Durante la misma es importante no olvidar la exploración de la columna cervical con el fin de descartar patología en este ámbito, ya sea concomitante o como responsable del proceso doloroso en forma de dolor referido. La exploración se completará con los arcos de movilidad, así como con una evaluación neurológica precisa.

La inspección constituirá el primer estadio de la exploración física. Con ella valoraremos el rictus del paciente, comparando la simetría y la uniformidad en los movimientos así como la normalidad de la marcha. El siguiente estadio lo constituirá la palpación, con la que verificaremos la normal posición de las articulaciones que componen el hombro, así como accidentes óseos como clavícula, surco bicipital, apófisis coracoides, troquín, troquíter y omoplato. El siguiente escalón llevará a la palpación de tejidos blandos, la cual podremos dividir en cuatro parcelas (7):

- Tendones del manguito de los rotadores. Punto de vital importancia pues la degeneración y desgarro de estos son alteraciones frecuentes en la población general, siendo causa frecuente de dolor en el hombro. El manguito rotador está compuesto por cuatro músculos: tres palpables en su inserción en el troquíter (supraespinoso el más superficial palpable en su inserción en el troquíter directamente por debajo del acromion, infraespinoso posterior al supraespinoso y el redondo menor inmediatamente posterior a los otros dos) y un cuarto, el subescapular (localizado por delante y que no es palpable) (7).

- Bolsa subacromial y subdeltoidea. La inflamación de esta bolsa es una patología frecuente que habitualmente origina importante dolor y restricción de la movilidad.

- Musculatura de la región. Deberá ser evaluada la consistencia de la musculatura, así como la presencia de contracturas focales con el posible reconocimiento de puntos gatillo. Entre los músculos que explorar estarán esternocleidomastoideo, pectoral mayor, bíceps, deltoides, trapecio, romboides menor y mayor y dorsal ancho.
- Arcos de movilidad (7). El rango de movimiento articular debe ser evaluado en todos sus planos. Existen una serie de pruebas especiales que nos orientarán sobre la localización específica de la lesión:

- Arco doloroso de Simons. Abducción activa de hombros. La aparición de dolor por encima de $60^{\circ}$ sugiere patología del manguito rotador.

- Test para evaluar la fuerza de los músculos infraespinoso y redondo menor. Con el hombro en $0^{\circ}$ o $90^{\circ}$ de abducción y el codo a $90^{\circ}$ se solicita la rotación externa resistida. La imposibilidad de realizarla o la aparición de dolor sugiere compromiso de estos músculos.

- Signo de Droppoff. Con el brazo en abducción de $90^{\circ}$ y el codo extendido realizará lenta aducción del mismo desencadenando una caída brusca de la extremidad, que indicará patología en el manguito.

- Test de Jobe. Test para evaluación del tendón del supraespinoso con el hombro en flexión de $90^{\circ}$, codo extendido y antebrazo en máxima pronación. En caso de dolor sugiere patología del tendón; en caso de incapacidad para mantener la posición deberemos sospechar rotura.

- Test de Gerber. Test para la evaluación del tendón del subescapular. Con el hombro en extensión y rotación interna, de manera que el dorso de la mano permanezca contra la región lumbar. Se le solicita al paciente que desplace la mano hacia atrás, contra resistencia. En caso de que sea capaz sugerirá la integridad del tendón.

- Test de Yergason. Evalúa el tendón bicipital. Con el hombro en aducción, codo en $90^{\circ}$ y antebrazo en pronación, se solicita una supinación resistida del antebrazo. La aparición de dolor en la cara anterior del hombro sugiere patología bicipital.

\section{Exploración neurológica}

Se compondrá de la evaluación de la fuerza de cada uno de los grupos musculares, pruebas de sensibilidad así como la verificación de la normalidad de los reflejos. Su alteración podrá orientar en ciertos casos hacia patología cervical.

\section{Pruebas complementarias (3)}

Las pruebas de laboratorio (determinación de marcadores inflamatorios, factor reumatoide...) se deberán considerar en aquellos pacientes con dolor persistente o en los que se sospechen afecciones de gravedad (10). Las pruebas radiológicas por rayos $\mathrm{X}$, ultrasonidos o resonancia magnética deberán ser consideradas cuando el dolor se prolongue 
en el tiempo. En caso de sospechar patología metastásica deberá realizarse una tomografía axial computarizada, así como una gammagrafía.

\section{TRATAMIENTO}

En general el tratamiento se iniciará con un abordaje conservador en el que haremos uso de antiinflamatorios no esteroideos y opioides menores asociados a terapia rehabilitadora, con el fin de evitar la atrofia muscular secundaria al dolor que complicarían el cuadro. El abordaje intervencionista será reservado a aquellos pacientes con patologías como capsulitis, síndrome de impingement, bursitis subacromial, artrosis acromioclavicular, patología glenohumeral (hombro congelado) y patología del manguito rotador (8).

\section{Infiltración "tres en uno" de la articulación del hom-} bro: glenohumeral, subacromial y acromioclavicular

La preferida por nuestro grupo de trabajo; con ella dispondremos de acceso a la articulación glenohumeral, articulación subacromial y acromioclavicular por una misma vía de abordaje, reduciendo así el grado de complicaciones y de disconfort del paciente.

\section{Técnica:}

Con el paciente en decúbito supino procederemos a identificar la zona correspondiente a la entrada de la articulación acromioclavicular. Tras infiltrar con anestésico local la zona de punción procederemos a la inserción de una aguja espinal 22G para alcanzar, en primer lugar, la articulación glenohumeral en profundidad. Tras verificar por medio de contraste la correcta posición de la aguja, se procederá a la infusión de 2,5 $\mathrm{ml}$ de una mezcla de anestésico local (AL) y corticoide particulado (CP) (nuestra mezcla habitual para los tres procedimientos es de $2 \mathrm{ml}$ de ropivacaína y $24 \mathrm{mg}$ de betametasona en $6 \mathrm{ml}$ ). En caso de dificultad hay que considerar la tracción de la extremidad afecta en dirección distal con el brazo inmediatamente adyacente y paralelo al tronco. Tras esto se procederá a abordar la articulación subacromial; para ello se retirará la aguja hasta el espacio subacromial, lugar en donde infundiremos $2,5 \mathrm{ml}$ de la mezcla de AL y CP previa verificación con contraste de la correcta posición de la aguja. En último lugar se procederá a la infiltración de la articulación acromioclavicular con el último ml de la mezcla (Fig. 1).

\section{Complicaciones:}

Aunque infrecuentes y reducidas en comparación con la realización por separado de cada una de ellas, las principales son la infección y las derivadas de la incorrecta infusión de corticoides particulados.

\section{Infiltración de la articulación glenohumeral (10)}

Con el paciente en decúbito supino o decúbito prono [en función de la vía de abordaje utilizado (supino cuando

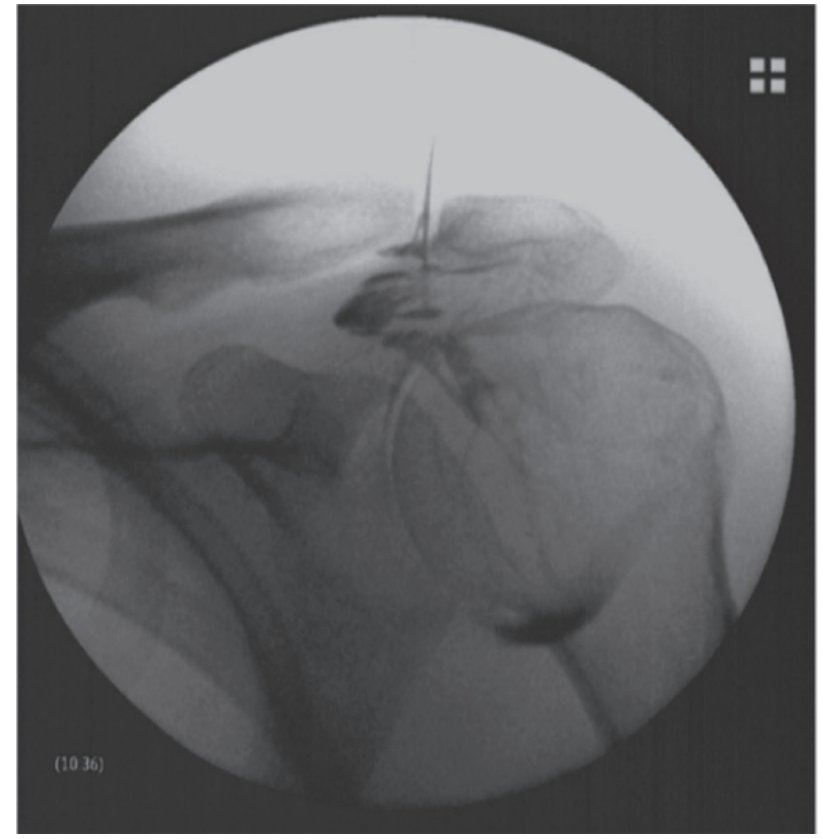

Fig. 1. Bloqueo 3 en 1.

el abordaje es anterior y prono en caso de que sea lateral o posterior)].

- Abordaje anterior: el punto de entrada se encuentra ligeramente lateral al punto intermedio entre el borde anterolateral del acromion y el proceso coracoideo.

- Abordaje lateral: con el paciente en decúbito prono con el brazo en rotación interna se palpará la articulación glenohumeral colocando los dedos entre el proceso coracoideo y la cabeza humeral; percibiremos el espacio articular como un surco lateral al proceso coracoideo. El punto de entrada será un dedo por debajo y lateral a la punta de la coracoides, permitiendo un avance lateral hasta la cavidad articular.

- Abordaje posterior: con el paciente en posición de decúbito prono; permitirá un acceso más sencillo a la articulación. El punto de entrada se localizará 2-3 cm inferior y $1 \mathrm{~cm}$ medial al borde posterolateral del acromion. Con este abordaje se corre el riesgo de lesionar el nervio supraescapular en caso de hacer un abordaje demasiado medial y el nervio axilar en caso de realizar un abordaje demasiado lateral.

- Abordaje superior: con el paciente en decúbito supino, con la cabeza en rotación contralateral al hombro afectado, se procederá a la localización mediante palpación de la articulación acromioclavicular; se verificará el punto de entrada a la misma con la ayuda de rayos $\mathrm{X}$ en dirección AP a la articulación. En este abordaje se alcanzará la articulación glenohumeral a través del espacio subacromial permitiendo el abordaje de este y de la acromioclavicular en un mismo tiempo (Fig. 2). 


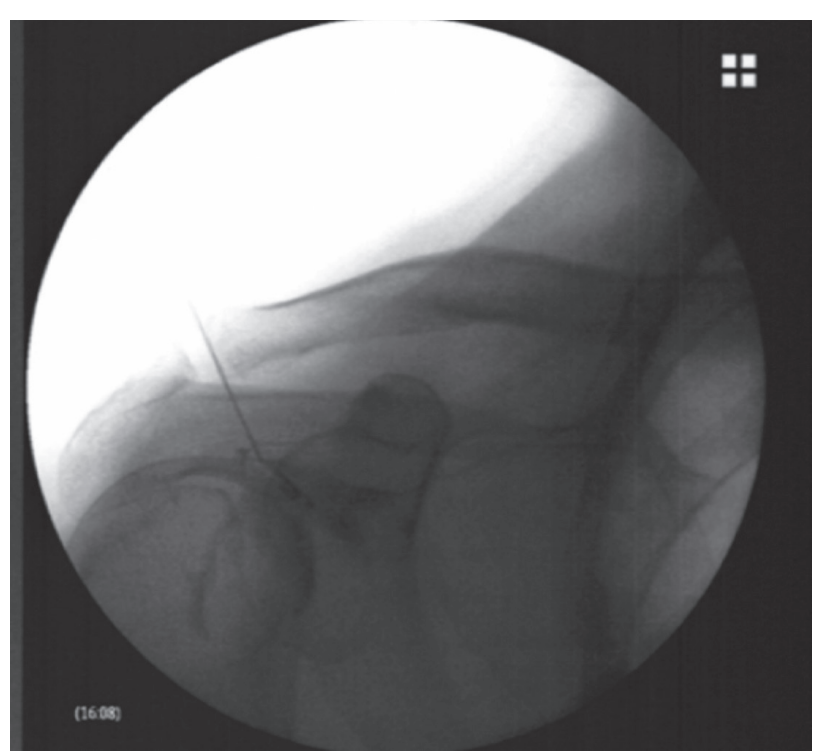

Fig. 2. Infiltración articulación glenohumeral.

*La artritis o inestabilidad de la articulación glenohumeral se caracteriza por sensación de inflamación alrededor del área subacromial. La inestabilidad glenohumeral es identificada por la laxitud de la cápsula y los ligamentos, que puede llevar a una subluxación del húmero (signo del ulcus) (10).

\section{Infiltración de la articulación acromioclavicular (12)}

Podrá ser realizada bajo guía fluoroscópica (la preferida por su capacidad para verificar una correcta difusión del contraste a través de la articulación) o ecográfica (permitirá una mayor versatilidad para su realización con un óptimo control de la difusión de la medicación infundida en la articulación). Describiremos la técnica bajo guía fluoroscópica por ser la realizada por nuestro grupo de trabajo; con el paciente en decúbito supino se procederá a localizar la zona entre la región anteriomedial del acromion y el límite distal de la clavícula. Tras desinfectar la zona con las medidas habituales y anestesiarla, se procederá a insertar una aguja de $22 \mathrm{G}$ en la zona posterior de la articulación acromioclavicular. A continuación se llevará a cabo la verificación de la correcta posición de la aguja con contraste para, una vez realizada, proceder a la infusión de $0,5 \mathrm{ml}$ de la mezcla de esteroides y AL (es importante advertir al paciente la posibilidad de la presencia de dolor en las 24-48 horas posteriores a la infiltración) (Fig. 3).

\section{Infiltración del espacio subacromial (11)}

La técnica podrá ser realizada con el paciente en decúbito prono o supino, con el brazo paralelo al cuerpo con el fin de facilitar el abordaje. El punto de entrada corresponderá a la prominencia ósea que se palpa a nivel anterolateral a la clavícula correspondiente a la coracoides. La entrada al espacio subacromial podrá realizarse desde un abordaje

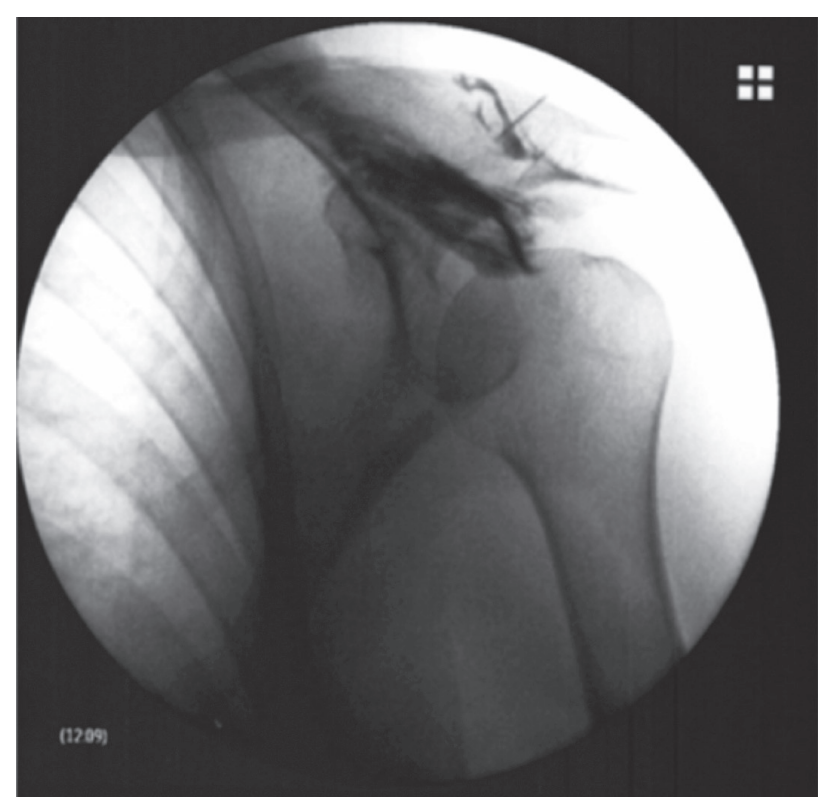

Fig. 3. Infiltración espacio acromioclavicular.

anterior, lateral y posterior. La elección dependerá de la patología y del hábito corporal del paciente.

- En el abordaje anterior la entrada al espacio subacromial se producirá directamente a la altura del ligamento coracoacromial. En él la aguja se dirigirá directa hacia el receso alrededor a la cabeza humeral posterior al acromion, horizontal directamente posterior al receso subacromial.

- Para el abordaje lateral servirá de referencia la identación existente por debajo del acromion, la aguja seguirá una dirección medial por debajo del acromion. Es habitualmente más complejo de alcanzar en pacientes obesos. Con este abordaje se puede acceder tanto al espacio subacromial como al manguito de los rotadores (Fig. 4).

- El abordaje posterior, por su parte, proporciona una entrada sencilla al espacio subacromial; sin embargo, la distancia que lo separa del ligamento coracoacromial disminuye su eficacia. El punto de entrada para este bordaje será inferior y medial al límite posterolateral del acromion. La aguja se dirigirá anteromedial con angulación superior hasta alcanzar la porción inferior del acromion en el espacio subacromial.

- El abordaje superior permite la entrada a través de la articulación acromioclavicular, proporcionando la posibilidad de acceso al resto de articulaciones, como hemos visto (Fig. 5).

Complicaciones:

- Hematoma e infusión intravascular de los fármacos secundario a la punción inadvertida de vasos axilares (13-15).

- Infección de la bursa subacromial (infrecuente). 


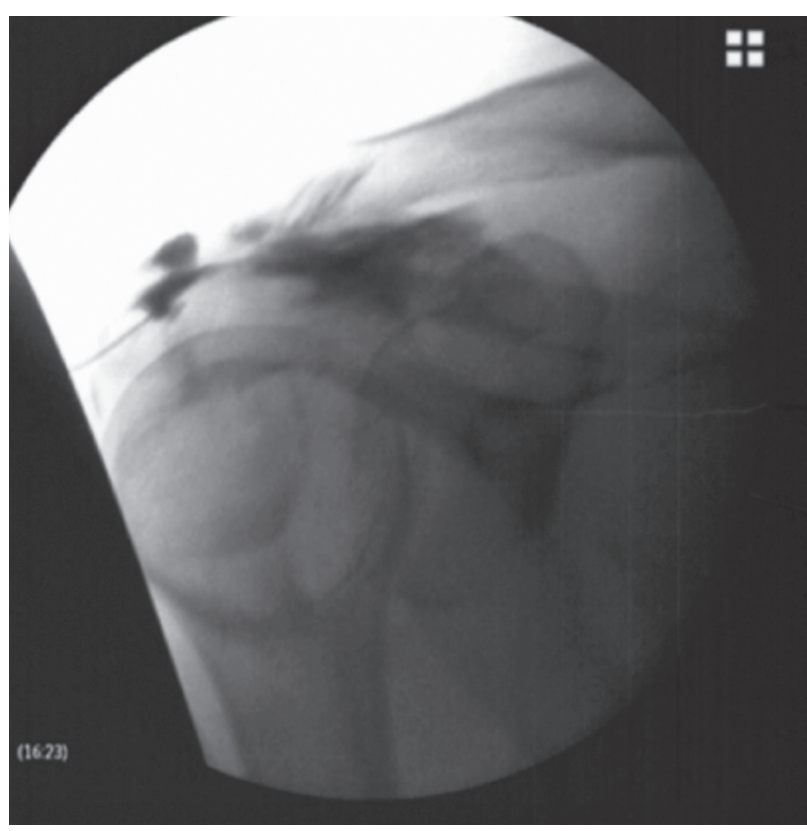

Fig. 4. Infiltración subacromial vía lateral.

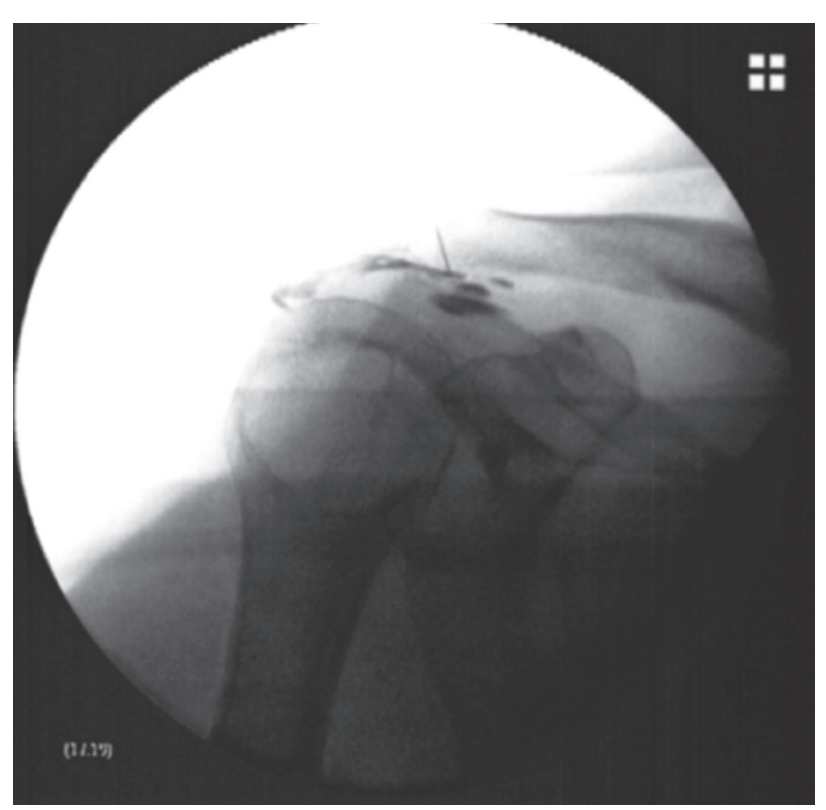

Fig. 5. Infiltración subacromial vía superior.

\section{Infiltración del tendón del biceps (11)}

Indicaciones:

- Tenosinovitis bicipital (pudiéndose abordar cualquiera de las dos porciones del tendón).

Esta puede surgir como consecuencia o asociado a un síndrome de impigement subacromial, manifestándose junto a tendinitis del manguito rotador e inestabilidad glenohumeral (13). Su presencia como síndrome primario suele surgir como consecuencia de un estrés repetitivo.
El cuadro clínico se manifiesta como dolor en la región anterior del hombro, que se incrementa con actividades como flexión del antebrazo sobre la cabeza, extensión del hombro así como flexión del codo. Presentan positividad para los test de Yergason's y Speed's (12). En caso de producirse la rotura del tendón largo se manifestará con el signo de Popeye. La subluxación del tendón bicipital se manifiesta como dolor en la región anterior del hombro asociado a un click y surge como consecuencia de la ruptura del ligamento trasverso humeral.

Técnica:

Existe la posibilidad de realizarlo con apoyo ecográfico o bien bajo guía fluoroscópica. Podrá ser realizada con el paciente en sedestación o preferentemente en decúbito supino (en caso de varones jóvenes) para prevenir la aparición de síndromes vasovagales.

Bajo guía fluoroscópica el punto de entrada corresponderá a la región superior del proceso coracoideo, la infiltración para la porción larga deberá ser realizada en el punto de máximo dolor en la corredera bicipital en la zona de la cabeza del húmero. Debe verificarse que la infusión de esteroide no es realizada en el espesor de la inserción del tendón, pues podría desencadenar en una ruptura del mismo.

Complicaciones:

- Infección (aunque es rara puede ocurrir) $(15,16)$.

\section{Infiltración del nervio supraescapular (11)}

\section{Indicación:}

Tratamiento de patologías del manguito de los rotadores, capsulitis adhesiva, artritis glenohumeral u hombro congelado. Se puede actuar sobre él por medio del bloqueo con esteroides y anestésico local o bien por medio de la variante pulsada de la radiofrecuencia, por ser una estructura neural mixta.

\section{Técnica:}

Se realizará con el paciente sentado o en decúbito prono. Como referencias anatómicas asumiremos la coracoides y el acromion. La técnica se podrá realizar con apoyo ecográfico o bien con apoyo de rayos X. La verificación de su localización se realizará por medio de la contracción de la musculatura del hombro.

- Rayos X: como referencia anatómica adoptaremos la escotadura supraescapular, se introducirá la aguja de neuroestimulación en dirección caudal y lateral hacia el borde lateral inferior de la escápula; la aguja se introducirá lentamente con la guía de los rayos $\mathrm{X}$ hasta verificar la respuesta de la neuroestimulación esperada (abducción y rotación externa, respuestas de los músculos supraespinoso e infraespinoso, respectivamente) (Fig. 6).

- Ecografía: se coloca la sonda lineal en posición trasversal sobre la fosa supraclavicular adyacente al acromion y a la espina de la escápula, de tal manera 


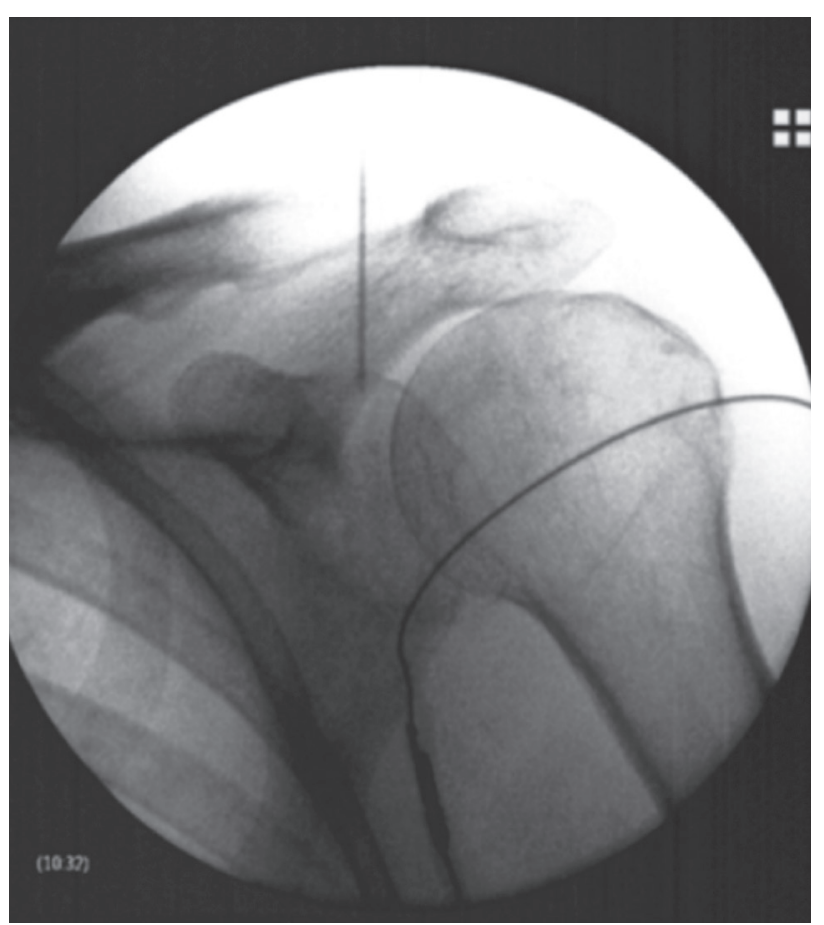

Fig. 6. Bloqueo nervio supraescapular.

que nuestro objetivo será alcanzar la región inferior al ligamento trasverso escapular, el cual encontraremos gracias a la presencia de la arteria supraescapular por encima del mismo. El nervio supraescapular en ocasiones es difícil de localizar (por su pequeño tamaño), situándose por debajo del ligamento trasverso en la región lateral.

\section{CORRESPONDENCIA:}

Jorge Orduña Valls

Clínica del Dolor

Centro Médico Teknon

Carrer de Vilana, 12

08022 Barcelona

e-mail: jorge.orduna@hotmail.com

\section{BIBLIOGRAFÍA}

1. Hoppenfeld S. Exploración física de la columna vertebral y las extremidades. México DF. El manual moderno. 1979.

2. Winters D. NHG-standaard schouderklachten. Huisarts. Wet 2008;51:555-65.

3. Huygen F, Patijn J, Rohof O, Lataster A, Mekhail N, Van Kleef M, et al. Painful Shoulder Complaints. In: VanZundert J, Patijn J, Hartrick CT, Lataster A, editors. Evidence Based Interventional Pain Medicine According to Clinical Diagnoses. New York: Wiley-Blackwell; 2012.

4. Viikari-Juntura E, Shiri R, Solovieva S, et al. Risk factors of aterosclerosis and shoulder pain is there an association? A systematic review. Eur J Pain 2008;12:412-26.

5. Kapandji IA. Fisiología articular. Tomo II. $5^{\text {a }}$ ed. Barcelona: Masson; 1997.

6. Navarro A, Alegre C; SECOT/SER. Monografías médico-quirúrgicas del aparato locomotor: el hombro. Barcelona: Masson; 2001.

7. Netter JC. Exploración clínica en ortopedia. Un enfoque para fisioterapeutas basado en la evidencia. Barcelona: Elsevier; 2006.

8. Oxner KG. MRI of the musculoskeletal system. Part 6. Clin Orthop \& Related Research 1997;334:354-73.

9. Mitchell C, Adebajo A, Hay E, Carr A. Shoulder pain diagnosis and management in primary care. BMJ 2005;331:1124-8.

10. Nosir HR. Chapter Upper Extremity Joint Injections. In: Manchikanti L, Singh V, editors. Interventional Techniques in Chronic Non-Spinal Pain. USA 2009. p. 377-90.

11. Fam AG. The shoulder. In: Fam AG, Lawry GV, Kreder HJ, editors. Musculoskeletal Examination and Joint Injection Techniques. Mosby Philadelphia: Elsevier; 2006. p. 12-24.

12. Baquie P. Joint and soft tissue injections. Aust Fam Physician 1996;25:1441-4.

13. Snider RK. Shoulder elbow, forearm, hand and wrist. In: Johnson RT, editor. Essentials of Musculoskeletal Care, American Academy of Orthopedic Surgeons. $4^{\text {th }}$ ed. 1999. p. $70-262$.

14. Gutiérrez G, Burroughs M, Poddar S. Clinical inquiries. Does injection of steroids and lidocaine in the shoulder relieve bursitis? J Fam Pract 2004;53:488-92.

15. Shah RV, Ericksen JJ, Lacerte M. Interventions in chronic pain management. New Frontiers: Invasive non-surgical interventions. Arch Phys Med Rehabil 2003;84(Supl. 1):S39-S44.

16. Almekinders LC, Temple JD. Etiology, diagnosis, and treatment of tendonitis: An analysis of the literature. Med Sci Sports Exerc 1998;30:1183-90. 